\title{
KOREAN LUNAR LANDER - CONCEPT STUDY FOR LANDING-SITE SELECTION FOR LUNAR RESOURCE EXPLORATION
}

\author{
Kyeong Ja Kimª, Christian Wöhler ${ }^{\mathrm{b}}$, Gwang Hyeok Juc , Seung-Ryeol Lee ${ }^{\mathrm{a}}$, Alexis P. Rodriguez ${ }^{\mathrm{d}}$, Alexey A. \\ Berezhnoy $^{\mathrm{e}}$, Stephan van Gasselt ${ }^{\mathrm{f}, \mathrm{g}}$ Arne Grumpe ${ }^{\mathrm{b}}$, Rabab Aymaz

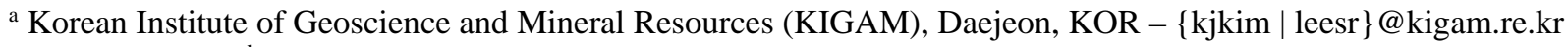 \\ b Technische Universität Dortmund, Image Analysis Group, Dortmund, GER - \\ \{christian.woehler | arne.grumpe | rabab.aymaz\}@tu-dortmund.de \\ c Korea Aerospace Research Institute (KARI), Daejeon, KOR - ghju@kari.re.kr \\ d Planetary Science Institute, Tucson, Arizona, US - alexis@psi.edu \\ e Sternberg Astronomical Institute, Moscow State University, Moscow, RUS - ber@sai.msu.ru \\ ${ }^{\mathrm{f}}$ Freie Universitaet Berlin, Dep. Earth Sci., Berlin, GER - stephan.vangasselt@fu-berlin.de \\ g University of Seoul, Department of Geoinformatics, Seoul, KOR
}

\section{Commission IV, WG IV/8}

KEY WORDS: Lunar Lander Mission, Landing Site Selection, Resource Exploration, Remote Sensing Data Analysis, Terrain Modelling, Feature Extraction

\begin{abstract}
:
As part of the national space promotion plan and presidential national agendas South Korea's institutes and agencies under the auspices of the Ministry of Science, Information and Communication Technology and Future Planning (MSIP) are currently developing a lunar mission package expected to reach Moon in 2020. While the officially approved Korean Pathfinder Lunar Orbiter (KPLO) is aimed at demonstrating technologies and monitoring the lunar environment from orbit, a lander - currently in pre-phase A - is being designed to explore the local geology with a particular focus on the detection and characterization of mineral resources. In addition to scientific and potential resource potentials, the selection of the landing-site will be partly constrained by engineering constraints imposed by payload and spacecraft layout. Given today's accumulated volume and quality of available data returned from the Moon's surface and from orbital observations, an identification of landing sites of potential interest and assessment of potential hazards can be more readily accomplished by generating synoptic snapshots through data integration. In order to achieve such a view on potential landing sites, higher level processing and derivation of data are required, which integrates their spatial context, with detailed topographic and geologic characterizations. We are currently assessing the possibility of using fuzzy c-means clustering algorithms as a way to perform (semi-) automated terrain characterizations of interest. This paper provides information and background on the national lunar lander program, reviews existing approaches - including methods and tools - for landing site analysis and hazard assessment, and discusses concepts to detect and investigate elemental abundances from orbit and the surface. This is achieved by making use of manual, semi-automated as well as fully-automated remote-sensing methods to demonstrate the applicability of analyses. By considering given boundary conditions, concrete procedures for determining potential landing sites of the Korean lunar lander could be proposed.
\end{abstract}

\section{INTRODUCTION}

As part of the national space promotion plan and presidential national agendas, South Korea's institutes and agencies under the auspices of the Ministry of Science, Information and Communication Technology and Future Planning (MSIP) are currently working on a phase-A study for a Korean Lunar Exploration Program (KLEP) (Ju et al, 2013). A Korean Pathfinder Lunar Orbiter (KPLO) is to be followed by a Korean Lunar Explorer (KLE) which constitutes an orbiter and a lander unit equipped with a small rover with a mass of approximately $20 \mathrm{~kg}$ (Fig. 1). Key goals of the Korean lunar mission are (1) investigations of lunar geology and space environment, (2) exploration of lunar resources, and (3) testing of future space and planetary exploration technology which will assist in future human activities on the Moon and beyond.

KPLO's main scientific return is considered to be composed of visual and spectral image data, space environmental measurements and data related to lunar resources. For the exploration of lunar resources two major exploration areas need to be considered: (1) resources in polar regions for the potential establishment of lunar bases or (2) future energy resources such as Helium-3 and precious rare earth elements along with radioactive resources like Uranium. KPLO is planned to operate in a circular polar orbit at $100 \mathrm{~km}$ altitude. Its size will be $1.9 \mathrm{x}$ 1.7 x $2.3(\mathrm{~m})$ with a dry mass of $550 \mathrm{~kg}$. The total science payload mass will amount to approximately $40 \mathrm{~kg}$ with instruments contributed by the Korean Aerospace Research Institute (KARI) and other Korean research institutes and centers, as well as NASA. The development periods for KPLO and KLE are considered to be 2016-2018 and 2017-2020, respectively (Ju et al, 2013).

To accomplish the main goals of the mission, a set of candidate lunar landing sites need to be considered. During the first three years, an investigation of prospective landing sites needs to be performed using not only the results of KPLO but also those from previous international lunar missions. The task of landing-site selection will comprise identification of (1) regions for potential resource exploration and (2) regions of scientific interest. Furthermore, a focus will be put on the identification of technologically feasible and safe landing areas under consideration of engineering constraints which will be imposed by mission, spacecraft and payload layout. While resource investigations have been prioritized, engineering studies and demonstrators are likely to play an additional integral role. 


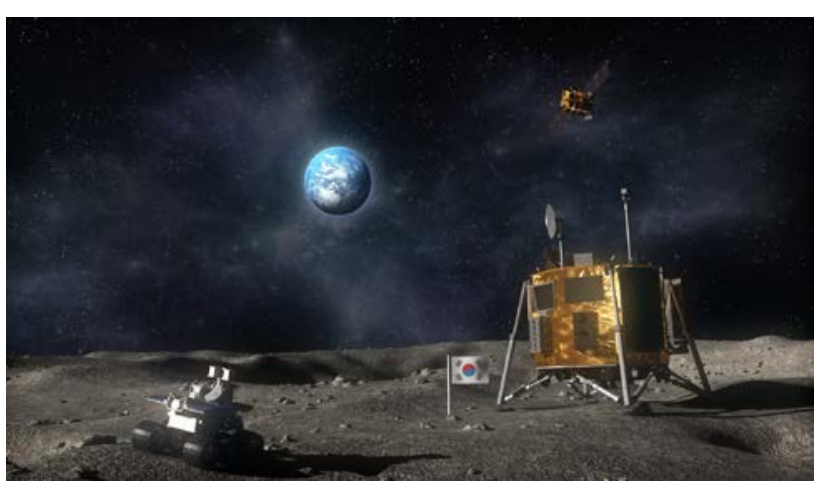

Figure 1. Depiction of the Korean rover, lander and orbiter in operation (source: KARI).

\section{METHODOLOGY}

\subsection{Remote sensing methods to be applied}

In order to perform investigations for prospective landing sites, derivation of auxiliary datasets and spatial data analyses are required using visual, multi- and hyperspectral data as well topography measurements. Investigations are then performed by (1) extraction of regional subsets based on high-level constraints, (2) identification of sites for potential resource exploration, (3) narrowing down operation sites based on low-level constraints, such as hazards.

High-level requirements imposed by engineering and mission constraints need to be investigated at smallest map scales first in order to extract regional and local subsets (e.g., time, altitudes, small-scale long-wavelength slope patterns, roughness types). These systematic and automatic analyses and evaluations (van Gasselt and Nass, 2013) are performed within information system environments using mostly (near-) global datasets that are publicly available and which may require additional coregistration work in case of residual misalignments.

Regional subsets will provide the settings for refined analyses at larger map scales which are based on science and resource criteria. These analyses are mainly conducted using stand-alone environments with dedicated routines and require visual interpretation and manual interaction. For the Korean mission package both mineralogical and elemental data need to be investigated as well as remote sending data associated with water resources such as neutron data.

The main step in identifying resource-bearing regions as potential landing sites is the analysis of orbital visual and nearinfrared spectral data sets, such as Clementine UVVIS and NIR multispectral image data (Nozette et al., 1994), Kaguya Multiband Imager (MI) data (Ohtake et al., 2008)], visual and NIR hyperspectral image data of the Chandrayaan-1 Moon Mineralogy Mapper $\left(M^{3}\right)$ (Pieters et al., 2009a) or NIR point spectrometer data of the Chandrayaan-1 SIR-2 instrument (Mall et al., 2009). A framework for the topographic and thermal correction of $\mathrm{M}^{3}$ hyperspectral image data and their normalization to a standard observation and illumination geometry is described in Wöhler et al. (2014), which can be used to routinely construct maps of the spectral reflectance, of the topography and of spectral parameters characterizing the absorption features near 1 and $2 \mu \mathrm{m}$ related to pyroxene (Bhatt et al., 2012) for arbitrary lunar regions. In combination with elemental abundance maps of low resolution e.g. obtained by the Lunar Prospector Gamma Ray Spectrometer (LP GRS) (Lawrence et al., 1998), the spectral parameter maps allow for the construction of high-resolution maps of the abundances of the main refractory elements $\mathrm{Al}, \mathrm{Mg}, \mathrm{Ca}, \mathrm{Fe}$ and $\mathrm{Ti}$ (Wöhler et al., 2014; Bhatt et al., 2015; Shkuratov et al., 2005). The strength of the absorption around $3 \mu \mathrm{m}$ related to hydroxyl $(\mathrm{OH})$ can also be mapped (Pieters et al., 2009b). $\mathrm{M}^{3}$ and SIR-2 data have been shown to be suitable for the detection of localized deposits of "exotic" minerals such as spinel or olivine which do not occur on large spatial scales (Mall et al., 2014). Spectral unmixing methods (Keshava and Mustard, 2002) can be used to estimate the constituent minerals of the surface material (e.g. orthopyroxene, clinopyroxene, plagioclase, ilmenite, olivine, and spinel) along with their fractional abundances based on the available orbital visual and NIR spectral data (Mall et al., 2012; Felder et al., 2013). Almost all variety of the known range of elemental composition of the lunar regolith can be explained by mixture of three end-members (ferroan anorthosite, mare basalt, and Mg-suite rocks) (Berezhnoy et al., 2005). For the search for rare mineralogies the technique of mapping distances of estimated composition from the three end-member plane has been shown to be powerful. In order to conduct such studies, high-resolution maps of abundances of $\mathrm{Fe}, \mathrm{Mg}$, and $\mathrm{Al}$ are needed (Berezhnoy et al., 2005).

Once candidate regions exhibiting resource-bearing minerals have been identified spectrally, they can be mapped topographically at high vertical and lateral resolution based on the combined stereo and intensity-based image analysis framework described in (Grumpe et al., 2014) applied to LRO Narrow Angle Camera (NAC) (Chin et al., 2007) images (Grumpe, 2015; Grumpe et al., 2015). This will also allow for a prediction of possible hazards e.g. due to boulders, steep slopes or impact craters, and include analyses for permanent shadows. This will further narrow down the selection of potential landings sites. A validation of such potential hazards for lander units will be conducted visually as well as semi-automatically (De Rosa et al., 2012). In this context, we are currently exploring the application of fuzzy c-means clustering algorithms (e.g. Miyamoto et al., 2008) for (partially) automatically detecting terrain types of high interest.

Example results obtained with some of the methods described in this section are shown in Section 3.

\subsection{Preferential potential landing sites}

Selection of landing sites on the Moon is a complex and challenging task, because various problems must be solved for successful soft landing and operation of a rover on the lunar surface. Among prospective landing sites are regions in the lunar polar regions as well as regions with anomalous mineralogical and elemental composition, such as high-abundance KREEP terrain or the South Pole-Aitken basin, and/or regions with confirmed high abundance of He-3 (Ju et al., 2013).

2.2.1 Lunar polar regions: In the lunar polar regions, several volatile compounds such as $\mathrm{CO}, \mathrm{H}_{2}, \mathrm{Ca}, \mathrm{Hg}, \mathrm{Mg}$ (Gladstone et al., 2010), $\mathrm{H}_{2} \mathrm{O}, \mathrm{H}_{2} \mathrm{~S}, \mathrm{NH}_{3}, \mathrm{SO}_{2}, \mathrm{C}_{2} \mathrm{H}_{4}, \mathrm{CO}_{2}, \mathrm{CH}_{3} \mathrm{OH}, \mathrm{CH}_{4}, \mathrm{OH}$ (Colaprete et al., 2010), and $\mathrm{Na}$ (Killen et al., 2010) were detected during the LCROSS impact experiment in the Cabeus crater. Majority of these species including water ice are present only at permanently shadowed lunar polar caps. However, there are several species which can be detected also at illuminated lunar polar regions.

Surface volatility temperature is defined as the temperature of evaporation with a rate of about $10^{-10} \mathrm{~cm} /$ year is estimated to be as $181 \mathrm{~K}, 226 \mathrm{~K}, 303 \mathrm{~K}$, and $378 \mathrm{~K}$ for $\mathrm{S}, \mathrm{Na}, \mathrm{Mg}$, and $\mathrm{Ca}$, respectively (Berezhnoy et al., 2012). Volatility temperature for deposits located at $2 \mathrm{~cm}$ depth is a little bit higher and equal to $202 \mathrm{~K}, 256 \mathrm{~K}, 342 \mathrm{~K}$, and $426 \mathrm{~K}$ for S, Na, Mg, and Ca, respectively (Berezhnoy et al., 2012). Thus, we expect the existence of deposits of the pure metals $\mathrm{Na}, \mathrm{Mg}$, and $\mathrm{Ca}$ in illuminated parts of lunar polar regions. Sulfur deposits can also 
be found, but only in selected coldest illuminated polar regions. Special techniques need to be developed for the detection of $\mathrm{S}$, $\mathrm{Na}, \mathrm{Mg}$, and $\mathrm{Ca}$ in elemental form in the polar lunar regolith.

$\mathrm{OH}$-containing minerals can also be found in illuminated polar regions. For detection of these minerals, remote sensing studies of the $3 \mu \mathrm{m} \mathrm{OH}$ band were already performed with the $\mathrm{M}^{3}$ instrument (Pieters et al., 2009a).

2.2.2 Regions with rare rock types: The analysis of Lunar Prospector data reveals presence of small-scale Ca-rich, Al-poor anomalies located at the lunar farside (Berezhnoy et al., 2005). Other interesting regions for improved on-site studies by rovers include aluminous basalts in the eastern part of Mare Frigoris (Kramer et al., 2015).

The thermal neutron enriched areas on the northern parts of the Moon were found to have very high Sm and Gd (Elphic et al., 2000). Interestingly, Change'-3 found Yttrium on the landing site soil (Peng et al., 2014). Regions with high contents of Ti-rich basalts are considered to be feasible from the viewpoint of utilization of lunar resources (Fa and Jin, 2007), as He-3 isotope content is correlated with regions of high Ti content (Busarev, 1994). Studies of Ti content at high spatial resolution will be helpful for understanding the correlation between main element abundances at resolutions of several km and element abundances at meter scale, which becomes important for arriving at an understanding about the practical utilization of lunar resources (Kim, 2016).

Other interesting regions with unusual mineralogical and elemental composition include central peaks of big craters such as crater Tycho, dark regions with impact melt deposits, and the South Pole Aitken basin.

Other regions of interest are, e.g., swirls that are often considered to form during recent cometary impacts (Bruck and Schultz, 2015). Studies of magnetic anomalies, regolith structure, and volatile content in swirls such as the Reiner-Gamma formation will be extremely helpful for explaining the origin of these structures. In situ studies by rovers of volatile compounds are important in the illuminated parts of the polar regions of the Moon $\left(80-90^{\circ} \mathrm{N}, 80-90^{\circ} \mathrm{S}\right)$, in the crater Aristarchus and at the locations of pyroclastic deposits.

Let us consider the technical limitations for performing successful operation of a lunar rover. Solar energy is required as a source of energy and solar illumination is required for performing studies of the lunar surface. Earth's visibility is required for successful exchange of information and remote operation of a rover by Earth-located scientists. It is difficult to see Earth and Sun simultaneously from the polar lunar regions. In contrast, landing at latitudes in the range between $70^{\circ} \mathrm{S}$ and $70^{\circ} \mathrm{N}$ is less difficult from this point of view.

For successful landing a rather flat surface is required. From this point of view, it would be difficult to land a rover in mountains and near central peaks of big craters. Landing on flat surfaces in mare regions (for example, in Mare Frigoris) and in selected highland regions will be possible. Let us note that increasing the accuracy of landing at a given point to $1-2 \mathrm{~km}$ will provide an opportunity to perform a successful soft landing even in regions with small hills and craters. Choosing a landing site with a small landing ellipse is based on the analysis of highresolution imagery and topographic data as well as mineralogical, elemental, and petrological maps of regions of interest.

At present, details of future Korean landing sites have not been published yet. However, it is considered to choose a landing site where new scientific discoveries are to be expected. The guidelines of NRC (NRC, 2007) or LEAG (Neal, 2009) will be considered as well as other countries' landing-site candidates. For example, the SELENE-2 Landing Site Research Board, established in March 2010 as a JAXA SELENE-2 pre-project team sub-group, received from 21 groups a number of 35 proposals describing more than 70 potential landing sites. A combination of the proposals according to a set of 10 potential landing sites was performed, which are Tycho, Apollo 14, Copernicus, Vertical Holes, Mare Humorum, Ina, Zucchius, Hansteen Alpha, Marius Hills, and Reiner Gamma (Saiki 2011). It is expected that Korea will organize its own landing-site research committee in order to select the most prospective landing site candidates. There will be two large aspects in selecting future landing site candidates which will largely depend on whether science goals focus on lunar geology or resource exploration.

The NRC defines a number of science topics that need to be addressed in the future (NRC, 2007), quoted here as follows:

1. The bombardment history of the inner solar system is uniquely revealed on the Moon.

2. The structure and composition of the lunar interior provide fundamental information on the evolution of a differentiated planetary body.

3. Key planetary processes are manifested in the diversity of lunar crustal rocks.

4. The lunar poles are special environments that may bear witness to the volatile flux over the latter part of solar system history.

5. Lunar volcanism provides a window into the thermal and compositional evolution of the Moon.

6. The Moon is an accessible laboratory for studying the impact process on planetary scales.

7. The Moon is a natural laboratory for regolith processes and weathering on anhydrous airless bodies.

8. Processes involved with the atmosphere and dust environment of the Moon are accessible for scientific study while the environment remains in a pristine state.

(NRC, 2007, p. 3). These topics are potentially to be addressed by the Korean mission. The Korean lunar program will also try to address as many of these fundamental research questions as possible. It is planned to set up a Korean Lunar Mapping Project for the first period to jointly work on definitions for science conduct and resource exploration. Once low-level engineering constraints will become available at required detail, targeted data analyses can be performed.

\section{EXAMPLE RESULTS}

An example of the construction of a LROC NAC-based highresolution DEM regards a small region of an impact melt deposit on the floor of the crater Korolev X (Denevi et al., 2012), which is shown in Figure 2. Figure 3 shows the NAC image data of the region for which the DEM has been constructed, Figure 4 depicts the DEM obtained using a shape-from-shading-based method (Grumpe et al., 2014; Grumpe and Wöhler, 2015), Figure 5 shows a DEM obtained based on stereo analysis of LROC NAC images $^{1}$, and Figure 6 displays a map of the surface slopes inferred from the high-resolution DEM. A comparison between Figures 4 and 5 reveals that the resolution of the shape-fromshading-based DEM is significantly higher than that of the stereo DEM and comes close to the resolution of the NAC image in Figure 3 used for constructing the DEM. Specifically, the narrow ridges and small craters visible in the NAC image are also visible in the high-resolution DEM, while most of them are not apparent 
in the stereo DEM. Close to the rim of the crater in the eastern part of the image, boulders sized between 1 and $2 \mathrm{~m}$ are visible. The slope map in Figure 6 indicates that for most of the region the slopes do not exceed values of 3-4 degrees, except for the walls of ridges and small craters. Such surface slope data are especially important for assessing the hazardousness of a potential landing site.

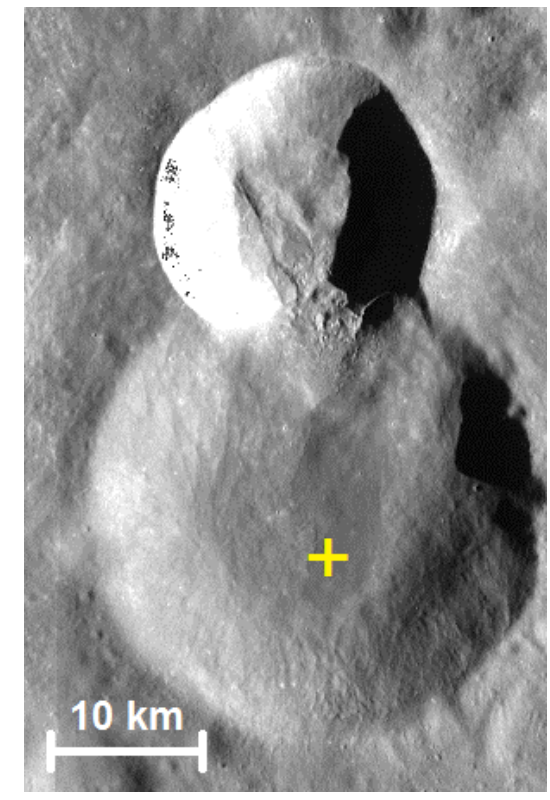

Figure 2. Lunar crater Korolev X (centered at $200.6^{\circ} \mathrm{E}$ and $0.6^{\circ} \mathrm{N}$ ), exhibiting an impact-melt deposit (dark region) on its floor. Excerpt from LROC WAC mosaic (Speyerer et al., 2011). The yellow cross marks the region of the high-resolution DEM presented in Figures 3-5.

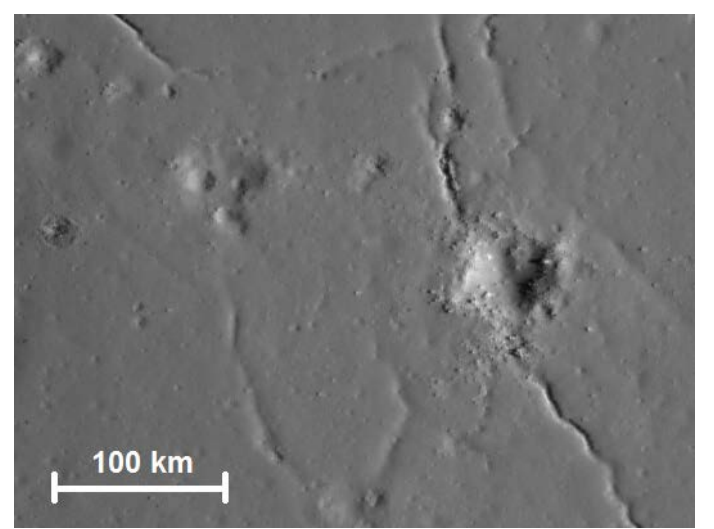

Figure 3. Excerpt from LROC NAC image M145664820R (downloaded from http://wms.lroc.asu.edu/lroc/view_lroc/LROL-LROC-2-EDR-V1.0/M145664820RE, image resolution is $0.62 \mathrm{~m}$ per pixel horizontal and $0.64 \mathrm{~m}$ per pixel vertical).

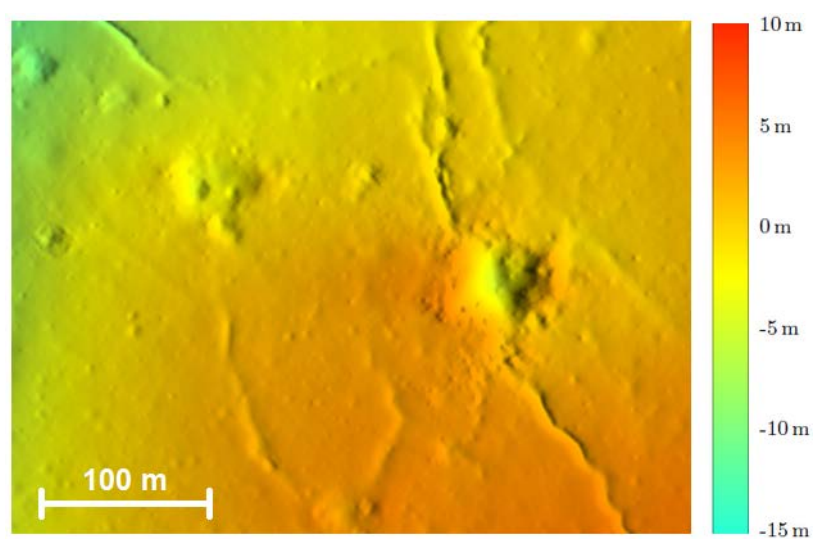

Figure 4. Shaded high-resolution DEM constructed using the shape-from-shading approach by Grumpe et al. (2014) and Grumpe and Wöhler (2015), combining GLD100 stereo data (Scholten et al., 2012) with LROC NAC reflectance data.

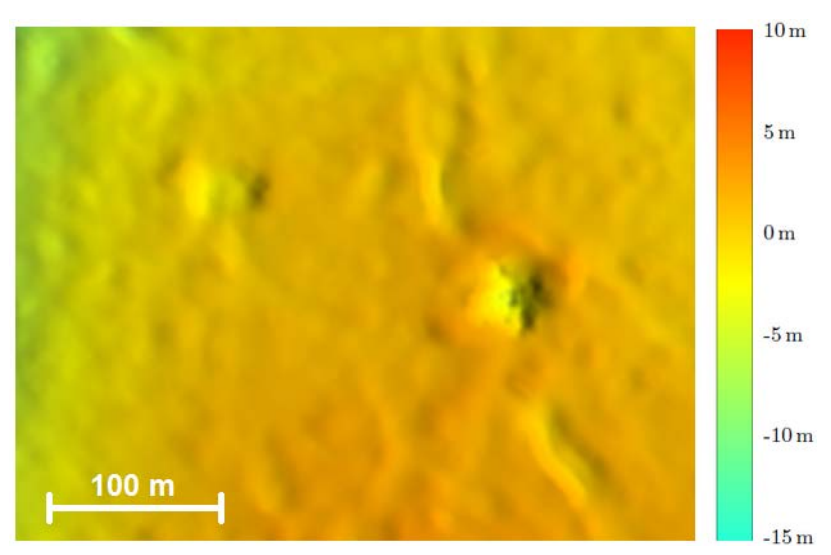

Figure 5. Shaded LROC NAC stereo DEM (data from http://wms.lroc.asu.edu//roc/view_rdr/NAC_DTM_IMPACTM ELT2).

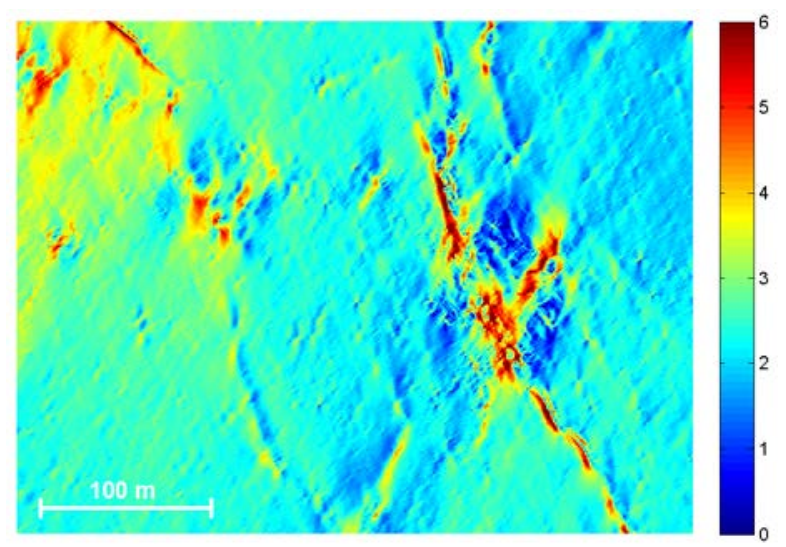

Figure 6. Map of the surface slope in degrees, inferred from the high-resolution DEM of Figure 4. 


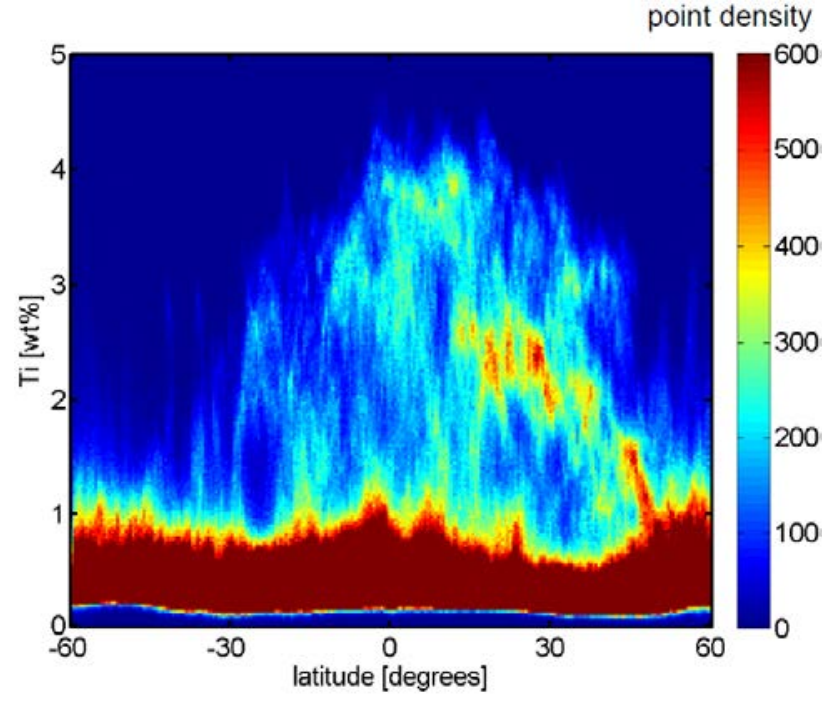

Figure 7. Latitudinal density plot of the lunar Ti distribution between $60^{\circ} \mathrm{S}$ (left) and $60^{\circ} \mathrm{N}$ (right). Each data point represents a grid cell of $0.05 \times 0.05$ degrees. Point density is measured within a $0.5^{\circ}$ latitude by $0.025 \mathrm{wt} \%$ neighbourhood. The highlands have Ti abundances below $1 \mathrm{wt} \%$, while clusters with Ti abundances of 2-4 wt $\%$ correspond to mare regions mainly on the northern nearside.

To illustrate elemental abundance mapping on the Moon, we used the approach of Wöhler et al. (2014) and Bhatt et al. (2015) described in Section 2.1, which relies on a combined analysis of $\mathrm{M}^{3}$ and LP GRS data. Figure 7 depicts the lunar Ti distribution as a latitudinal density plot derived from a nearly global $\mathrm{Ti}$ abundance map obtained using the method proposed by Bhatt et al. (2015). It shows that most mare basalts have a relatively low Ti content of 2-3 wt\%, with only small regions reaching values of $4 \mathrm{wt} \%$ and more. These Ti-rich mare basalts preferentially occur on the northern hemisphere and are virtually absent on the southern hemisphere. The area in the plot with Ti abundances below 1 wt\% corresponds to lunar highlands. Figure 8 shows a $\mathrm{Ti}$ abundance map of 20 pixels per degree resolution obtained with the same approach. It covers a region in eastern Oceanus Procellarum of about 300 by $600 \mathrm{~km}$ size which clearly shows mare basalts of strongly different Ti content, including regions with $\mathrm{Ti}$ abundances of more than $5 \mathrm{wt} \%$ that might be attractive as potential landing sites as pointed out in Section 2.2. Similar abundance maps of the elements $\mathrm{Al}, \mathrm{Mg}, \mathrm{Ca}, \mathrm{Fe}$ and $\mathrm{Ti}$ can be constructed for any potential landing site region at the full $\mathrm{M}^{3}$ resolution (corresponding to 140 meters per pixel, see Pieters et al., 2009a) to assess their scientific relevance.

Nearly global maps of the modal abundances of the minerals plagioclase and pyroxene are shown in Figure 9. To obtain these maps, a clustering of the $\mathrm{M}^{3}$ spectra on a nearly global mosaic of 20 pixels per degree resolution into a set of seven prototype spectra was performed (Grumpe and Wöhler, 2014). As a catalogue of potential endmembers for spectral unmixing, 19 spectra of lunar mare and highland samples published by the Lunar Soil Characterization Consortium (Pieters et al., 2000; Taylor et al., 2001; download of reflectance spectra and compositional analyses from http://www.planetary.brown.edu/ relabdocs/LSCCsoil.html and http://web.utk.edu/ pgi/data.html, respectively) were used, where we used the data of the 20-45 $\mu \mathrm{m}$ grain size fraction. Spectral unmixing was performed for each of the seven prototype spectra using the computationally expensive genetic algorithm-based approach of Felder et al. (2013) in order to determine for each prototype spectrum out of which of the 19 potential endmembers it is composed. We constructed a nearly

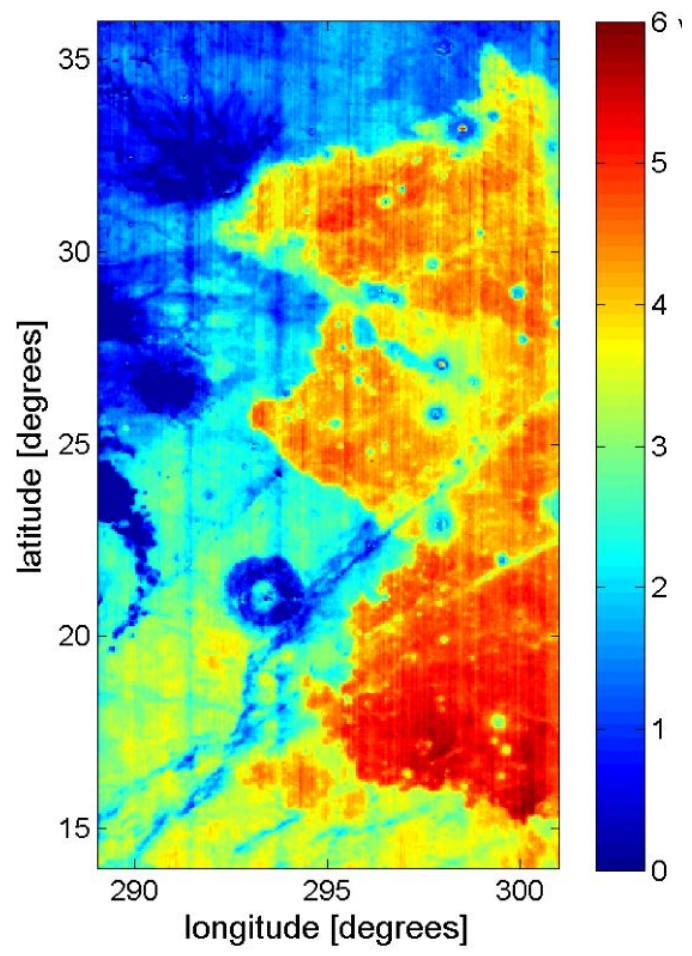

Figure 8. Ti abundance map (weight-\%) of 20 pixels per degree resolution of a part of western Oceanus Procellarum, showing mare basalts of low and high Ti content. Map constructed based on LP GRS data and $\mathrm{M}^{3}$ hyperspectral image data using the method described in Bhatt et al. (2015).

global $\mathrm{M}^{3}$ mosaic resampled to 10 pixels per degree and corrected for topography using GLD100 data (Scholten et al., 2012) and for thermal emission using the method of Wöhler et al. (2014). Each pixel spectrum of this mosaic was assigned to the most similar of the seven prototype spectra. Then, using the corresponding prototype-specific set of endmembers, nonlinear spectral unmixing according to the Hapke model (Hapke, 1984, 2002) was performed in order to estimate the relative fractions of the endmember spectra. Mathematically, this nonlinear unmixing procedure can be treated as a linear unmixing in terms of the single-scattering albedo (Dhingra et al., 2011), which reduces the computational burden such that large amounts of hyperspectral data can be processed. Based on the available LSCC compositional analyses stating the modal abundances of a variety of mineral species (http://web.utk.edu/ pgi/data.html), the endmember fractions estimated for each pixel of the $\mathrm{M}^{3}$ mosaic were converted into mineral fractions. The obtained nearly global maps of the minerals plagioclase and pyroxene (total) are shown in Figure 9. The plagioclase map mainly shows the general compositional difference between lunar mare and highland areas, while the pyroxene map reveals mare basalt areas of different composition.

Similar mineral maps can be constructed for a larger variety of mineral species for arbitrary potential landing sites at full $\mathrm{M}^{3}$ resolution in order to identify regions of high scientific interest.

\section{SUMMARY AND CONCLUSION}

In this work we have given an overview of the planned Korean Lunar Exploration Program (KLEP) involving two orbiters and a lander unit equipped with a rover. We have discussed the general properties of suitable landing sites, especially regarding the occurrence of volatiles and/or rare minerals, and we have presented methods for the identification of landing sites which 


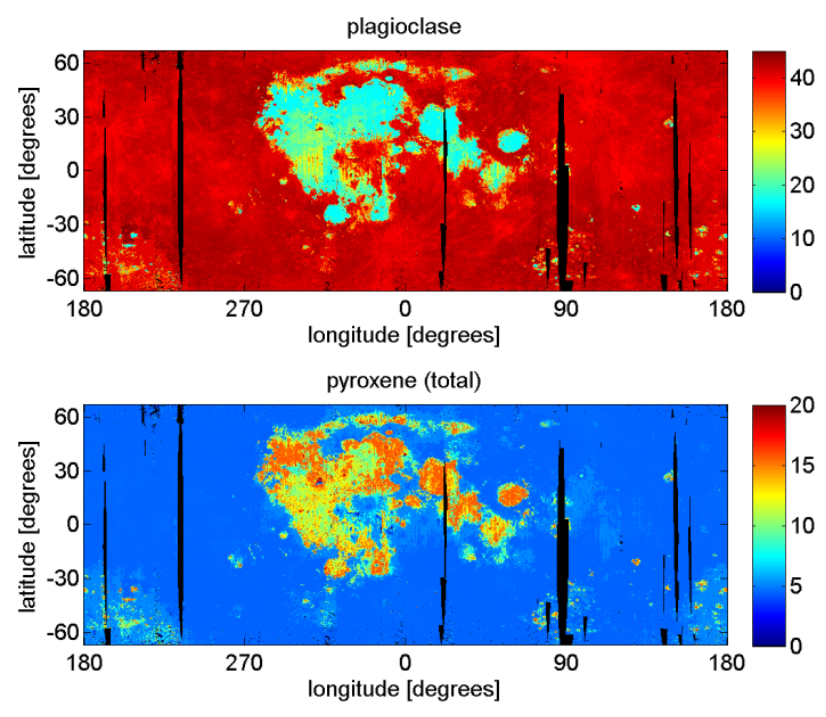

Figure 9. Fractional abundance maps (in percent) of the mineral classes plagioclase and pyroxene (total). Black denotes missing data.

are safe under consideration of engineering constraints and at the same time suitable for resource investigation. An important approach to the identification of resource-bearing regions is the analysis of multispectral and hyperspectral data.

We have described how elemental abundance maps can be obtained by combined analysis of LP GRS and $\mathrm{M}^{3}$ data, and how the approach of spectral unmixing allows for the construction of mineral abundance maps. Furthermore, possible hazards e.g. due to boulders, steep slopes or impact craters can be analysed relying on DEMs constructed from high-resolution orbital image data using shape from shading based techniques. Such DEMs can also be used for identifying permanently shadowed areas in the polar regions, which might also pose a risk to a lander. Exemplary results of these remote sensing methods have been presented. They can all be routinely applied to arbitrary potential landing site regions.

The next step consists of initiating a Korean Lunar Mapping Project to examine scientific aspects as well as factors relevant for resource extraction. With the availability of detailed low-level engineering constraints, more selective analyses of specific landing site candidates will be performed.

\section{ACKNOWLEDGEMENTS}

This project was partially supported by KIGAM research program (16-3116) and Space Core Technology Development Program (NRF-2015-M1A3A3-A030-27286)/(15-6502) funded by the Ministry of Science, ICT and Future Planning.

\section{REFERENCES}

Berezhnoy, A. A., Hasebe, N., Kobayashi M., Michael, G. G., Okudaira, O., Yamashita, N., 2005. A three end-member model for petrologic analysis of Lunar Prospector gamma-ray spectrometer data. Planet. Sp. Sci., 53, pp. 1097-1108

Berezhnoy, A. A., Kozlova, E. A., Sinitsyn, M. P., Shangaraev, A. A., Shevchenko, V. V., 2012. Origin and stability of lunar polar volatiles. Adv. Space Res., 50(12), 1638-1646.

Bhatt, M., Mall, U., Bugiolacchi, R., McKenna-Lawlor, S., Banaszkiewicz, M., Nathues, A., Ullaland, K., 2012. Lunar iron abundance determination using the 2- $\mu \mathrm{m}$ absorption band parameters. Icarus, 220, pp. 51-64

Bhatt, M., Mall, U., Wöhler, C., Grumpe, A., Bugiolacchi, R., 2015. A comparative study of iron abundance estimation methods: Application to the western nearside of the Moon. Icarus, 248, pp. 72-88

Bruck, S. M., Schultz, P. H., 2015. Cometary impact effects at the Moon: Implications for lunar swirl formation, Icarus, 257, pp. 194-206

Busarev, V. V., 1994. On problems of search of some lunar potential resources by spectral methods, Astronomicheskij Vestnik, 28(2), pp. 47-58

Chin, G. and 16 coauthors, 2007. Lunar Reconnaissance Orbiter Overview: The Instrument Suite and Mission. Space Sci. Rev., 129(4), pp. 391-419

Colaprete, A. and 16 coauthors, 2010. Detection of Water in the LCROSS Ejecta Plume. Science, 330(6003), pp. 463-468

Denevi, B. W., Koeber, S. D., Robinson, M. S., Garry, W. B., Hawke, B. R., Tran, T. N., Lawrence, S. J., Keszthelyi, L. P., Barnouin, O. S., Ernst, C. M., Tornabene, L. L., 2012. Physical constraints on impact melt properties from Lunar Reconnaissance Orbiter Camera images. Icarus, 219, pp. 665-675

Dhingra, D., Mustard, J. F., Wiseman, S., Pariente, M., Pieters, C. M., Isaacson, P. J., 2011. Non-linear Spectral Un-mixing Using Hapke Modeling: Application to Remotely Acquired M3 Spectra of Spinel Bearing Lithologies on the Moon. LPSC XXXXII, abstract \#2431.

Elphic, R. C., Lawrence, D. J., Feldman, W. C., Barraclough, B. L., Maurice, S., Binder, A. B., Lucey, P. G., 2000. Lunar rare earth element distribution and ramifications for $\mathrm{FeO}$ and $\mathrm{TiO}_{2}$ : Lunar Prospector neutron spectrometer observations. J. Geophys. Res., 105(E8), pp. 20333-20345

Fa, W., Jin, Y.-Q., 2007. Quantitative estimation of helium-3 spatial distribution in the lunar regolith layer. Icarus, 190, pp. 1523

Felder, M.P., Grumpe, A., Wöhler, C., Mall, U., 2013. Automated Endmember Selection for Nonlinear Unmixing of Lunar Spectra. European Planetary Science Congress, 8, EPSC2013-684-3

van Gasselt, S., Nass, A., 2013. On the Applicability of Geographic Information Systems for Landing-Site Assessments. Europ. Planet. Sci. Conf., 8, EPSC2013-1001

Gladstone, G.R. and 19 coauthors, 2010. LRO-LAMP Observations of the LCROSS Impact Plume. Science, 330(6003), pp. 472-476

Grumpe, A., 2015. Integrated recovery of elevation and photometric reflectance properties from hyperspectral data. Ph. D. thesis, TU Dortmund, http://dx.doi.org/10.17877/ DE290R-16479

Grumpe, A., Belkhir, F., Wöhler, C., 2014. Construction of lunar DEMs based on reflectance modeling. Adv. Sp. Res., 53(12), pp. 1735-1767

Grumpe, A., Wöhler, C., 2014. Automatic segmentation of petrographic geologic units based on elemental abundance maps. European Lunar Symposium, London, UK. 
Grumpe, A., Wöhler, C., Liu, M., Wu, B., 2015. Construction of Very High Resolution DTMs Based on NAC Images Using Stereo Analysis and Shape from Shading: A First Glance, LPSC XXXXVI, abstract \#1787

Hapke, B., 1984. Bidirectional reflectance spectroscopy: 3. Correction for macroscopic roughness. Icarus, 59(1), pp. 41-59.

Hapke, B., 2002. Bidirectional reflectance spectroscopy: 5. The coherent backscatter opposition effect and anisotropic scattering. Icarus, 157(2), pp. 523-534

Ju, G.-H., Bae, J., Choi, S.-J., Lee, W. B., Lee, C. J., 2013. New Korean Lunar Exploration Program (KLEP): An Introduction to the Objectives, Approach, Architecture, and Analytical Results. Int. Astron. Congress, \#IAC-13,A3,2C,10,x19392

Keshava, N., Mustard, J.F., 2002. Spectral unmixing, IEEE Signal Proc. Mag., 19(1), pp. 44-57

Killen, R. M., Potter, A. E., Hurley, D. M., Plymate, C., Naidu, S., 2010. Observations of the lunar impact plume from the LCROSS event. Geophys. Res. Lett., 37(23), DOI: 10.1029/ 2010GL045508

Kim, K. J., Wöhler, C., Hasebe, N., van Gasselt, S., Berezhnoy, A. A., Rodriguez, J. A. P., Grumpe, A., 2016. Lunar silicon distribution as observed by the Kaguya gamma-ray spectrometer and Chandrayaan-1 Moon Mineralogy Mapper ( $\left.\mathrm{M}^{3}\right)$ calibration. LPSC XXXXVII, abstract \#1473.

Kramer, G. Y., Jaiswal, B., Hawke, B. R., Öhman, T., Giguere, T. A., Johnson, K., 2015. The basalts of Mare Frigoris. J. Geophys. Res., 120(10), pp. 1646-1670, DOI: 10.1002/ 2014JE004753

Lawrence, D. J., Feldman, W. C., Barraclough, B. L., Binder, A. B., Elphic, R. C., Maurice, S., Thomsen, D. R., 1998. Global Elemental Maps of the Moon: The Lunar Prospector Gamma-Ray Spectrometer. Science, 281(5382), pp. 1484-1489. Data download from http://www.mapaplanet.org/explorer/moon.html

Mall, U., Banaszkiewicz, M., Brønstad, K., McKenna-Lawlor, S., Nathues, A., Soraas, F., Vilenius, E., Ullaland, K., 2009. Near Infrared Spectrometer SIR-2 on Chandrayaan-1. Current Science, 96(4), pp. 506-511

Mall, U., Korokhin, V., Bugiolacchi, R., Shkuratov, Y., 2012. Towards a Quantitative Determination of the Modal Mineralogy of Planetary Surfaces Using Near-Infrared Spectroscopic Data from the Moon. LPSC XXXXIII, abstract \#1893

Mall, U., Wöhler, C.; Grumpe, A. et al., 2014. Characterization of lunar soils through spectral features extraction in the NIR. Adv. Sp. Res., 54(10), pp. 2029-2040

Miyamoto, S., Ichihashi, H., Honda, K., 2008. Algorithms for Fuzzy Clustering. Methods in c-Means Clustering with Applications. Studies in Fuzziness and Soft Computing, vol. 229, Springer-Verlag Berlin Heidelberg

Neal, C. R., 2009. The Moon 35 years after Apollo: What's left to learn? Chemie der Erde - Geochemistry, 69(1), pp. 3-43

Nozette, S. and 34 coauthors, 1994. The Clementine mission to the Moon: Scientific overview. Science, 266, pp. 1835-1839

Ohtake, M., Haruyama, J., Matsunaga, T., Yokota, Y., Morota, T., Honda, C., and LISM team, 2008. Performance and scientific objectives of the SELENE (KAGUYA) Multiband Imager. Earth Planets Space, 60, pp. 257-264

NRC, 2007. The Scientific Context for Exploration of the Moon, Final Report, 120 pp., National Research Council, Nationa Academies Press.

Peng, W. X. and 18 coauthors, 2014. Active Particle-Induced XRay Spectrometer for Chang'e-3 YuTu Rover Mission and its First Results. LPSC XXXXV, abstract \#1699

Pieters, C.M. and 19 coauthors, 2009a. The Moon Mineralogy Mapper $\left(\mathrm{M}^{3}\right)$ on Chandrayaan-1. Current Science, 96(4), pp. 500505. Data download from http://pds-imaging.jpl.nasa.gov/ volumes/m3.html

Pieters, C.M. and 28 coauthors, 2009b. Character and Spatial Distribution of $\mathrm{OH} / \mathrm{H}_{2} \mathrm{O}$ on the Surface of the Moon Seen by $\mathrm{M}^{3}$ on Chandrayaan-1. Science, 326, pp. 568-572

Pieters, C. M., Taylor, L. A., McKay, D. S., Wentworth, S., Morris, R. V., Keller, L. P., 2000. Spectral characterization of lunar mare soils. LPSC XXXI, abstract \#1865

De Rosa, D., Bussey, B., Cahill, J. T., Lutz, T., Crawford, I. A., Hackwill, T., van Gasselt, S., Neukum, G., Witte, L., McGovern, A., Grindrod, P. M., Carpenter, J., 2012. Characterisation of potential landing sites for the European Space Agency's Lunar Lander project. Planet. Sp. Sci., 74(1), pp. 224-246

Saiki, K., Arai, T., Araki, H., Ishihara, Y., Ohtake, M., Yuzuru, K., Kobayashi, N., Sugihara, T., Haruyama, J., Honda, C., Sato, H., 2011. Landing Site Evaluation for the Next Lunar Exploration Project: SELENE-2. American Geophysical Union, Fall Meeting, abstract \#P43B-1681

Scholten, F., Oberst, J., Matz, K.-D., Roatsch, T., Wählisch, M., Speyerer, E. J., Robinson, M. S., 2012. GLD100: The near-global lunar 100 m raster DTM from LROC WAC stereo image data. $J$. Geophys. Res., 117, DOI: 10.1029/2011JE003926. Data download from http://wms.lroc.asu.edu/lroc/view_rdr/ WAC_GLD100

Shkuratov, Y.G., Kaydash, V.G., Stankevich, D.G., Starukhina, L. V., Pinet, P. C., Chevrel, S. D., Daydou, Y. H., 2005. Derivation of elemental abundance maps at intermediate resolution from optical interpolation of Lunar Prospector gammaray spectrometer data. Planet. Sp. Sci., 53(12), pp. 1287-1301

Speyerer, E. J., Robinson, M. S., Denevi, B. W., LROC science team, 2011. Lunar Reconnaissance Orbiter camera global morphological map of the Moon. LPSC XXXXII, abstract \#2387. Data download from http://wms.lroc.asu.edu/lroc/view_rdr/ WAC_GLOBAL

Taylor, L. A., Pieters, C. M., Morris, R. V., Keller, L. P., McKay, D. S., 2001. Lunar mare soils: Space weathering and the major effects of surface-correlated nanophase Fe. J. Geophys. Res., 106 (E11), 27,985-28,000

Wöhler, C., Grumpe, A., Berezhnoy A. A., Bhatt, M. U., Mall, U., 2014. Integrated topographic, photometric and spectral analysis of the lunar surface: Application to impact melt flows and ponds. Icarus, 235, pp. 86-122 